Research Article

\title{
Preparation of Polymeric Nanomaterials Using Emulsion Polymerization
}

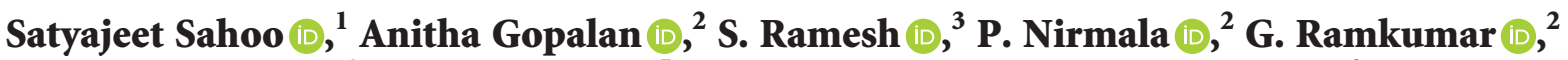 \\ S. Agnes Shifani $\left({ }^{4},{ }^{4}\right.$ Ram Subbiah $\left(1,{ }^{5}\right.$ and J. Isaac JoshuaRamesh Lalvani $\oplus^{6}$ \\ ${ }^{1}$ Department of Electronics and Communication Engineering, \\ Vignan's Foundation for Science,Technology and Research (Deemed to be University), Vadlamudi, Guntur, \\ Andra Pradesh 522213, India \\ ${ }^{2}$ Department of Electronics and Communication Engineering, Saveetha School of Engineering, SIMATS, Chennai 602105, \\ Tamil Nadu, India \\ ${ }^{3}$ Department of Electronics and Communication Engineering, Sri Shakthi Institute of Engineering and Technology, \\ Coimbatore 641062, Tamilnadu, India \\ ${ }^{4}$ Department of Electronics and Communication Engineering, Jeppiaar Maamallan Engineering College, \\ Chennai, Tamilnadu, India \\ ${ }^{5}$ Department of Mechanical Engineering, Gokaraju Rangaraju Institute of Engineering and Technology, \\ Nizampet, Hyderabad, India \\ ${ }^{6}$ Department of Mechanical Engineering, Faculty of Mechanical and Production Engineering, AMIT, Arba Minch University, \\ Arba Minch, Ethiopia
}

Correspondence should be addressed to Satyajeet Sahoo; satyajeetsahoo.eltc@gmail.com and J. Isaac JoshuaRamesh Lalvani; isaac.jrl@amu.edu.et

Received 24 August 2021; Accepted 23 September 2021; Published 8 October 2021

Academic Editor: P Ganeshan

Copyright (c) 2021 Satyajeet Sahoo et al. This is an open access article distributed under the Creative Commons Attribution License, which permits unrestricted use, distribution, and reproduction in any medium, provided the original work is properly cited.

\begin{abstract}
Nanoparticles are said to be active particles which are entrapped in the surface of the polymeric core. Since nanoparticles were used in medical and biotechnological fields, there is a great demand in the preparation of nanoparticles. Nanoparticles are prepared from different substances; mainly, polymer material is used in the field of preparing nanomaterials. There are different methods involved in the preparation of nanoparticles from the polymer. Various experiments and research studies were carried out on the basic preparation of nanoparticles. Emulsion polymerization could be used to make polymeric nanoparticles with a high solid concentration without the need of surfactants. To make carboxylate polystyrene beads or amidine polystyrene nanoparticles, polymeric nanocolloids containing surface functional groups were produced. In this research, the preparation of nanoparticles from emulsion polymerization is represented along with the size and distribution material.
\end{abstract}

\section{Introduction}

To achieve the challenges in drug delivery, polymer nanotechnology is considered as one of the promising systems. This involves drug targeting as well as delivering undelivered compounds such as oligonucleotides. The important challenges in nanotechnology are the preparation of nanoparticles, with suitable properties of nanoparticles, and this confirms proper drug transporting and pointing [1]. Various grades of poly and copolymers are used to prepare the nanoparticles in a wide range. The ability to synthesize polymers with well-controlled structures and conformation also paves the way for nanoparticles with precisely tuned characteristics, which are needed to meet drug targeting goals. This enthusiasm stems from the belief that nanoparticles with sizes ranging 
from 10 nanometers to more than 100 nanometers improved chemical, physical, or biological capabilities while developing specifically for certain applications [2].

Water-in-oil (W/O) or reverse emulsion polymerization is a suitable and well-studied technique for the generation of water-soluble nanopolymer particles with high molecular weight and low viscosity. The important parameter for the inverse emulsion polymerization process is established by the originated type and amount and the emulsifier type. Various solvents were utilized as a continuous phase, reaction locus, and monomeric reservoir in the reverse emulsion polymerization of acrylamide. The effect of the process parameter is determined, and hydrophilic equilibrium, emulsifier type and concentration, initiator, and reaction temperature are just some of the questions that have been investigated.

Microfluidics capacity to quickly mix chemicals, produce the homogeneous reaction, continually modify reaction environments, and introduce chemical components at exact time intervals during reaction advancement has made it popular knowledge for a wide range of applications; reagents are utilized in very modest concentrations [3]. By allowing the fabrication of the inorganic nanoparticle, the combination of the microfluid and particle technology is obtained. The inorganic nanoparticle is about $4-9 \mathrm{~nm}$ and the microparticle is $10 \mathrm{~nm}$, facing a significant issue for its maximum exploitation in certain circumstances with narrow size dispersal or different morphologies. This is especially relevant because the bulk mixing and nanoprecipitation of medicines and biodegradable polymeric predecessors used to make biodegradable polymeric nanoparticles often gets out of control, which can affect the characteristics of resultant nanoparticles. Microfluidics with quick and customizable mixing allows for greater control of the nanoprecipitation procedure as well as screening of diverse preparation circumstances on a single platform by altering parameters such as precursor composition, flow rates, and mixing time [4].

Polymerization can be used to produce live block copolymers separately or in combination with the stage of micelle formation. The thermodynamic properties of these polymer/solvent devices allow the development of nanoparticles of different shapes such as spheres and cords. Additional steps can be performed during block copolymer synthesis or in one or more posttreatment procedures performed on the nanoparticles produced to introduce special properties such as functional groups and water generation. At least two alternative approaches can be used in the manufacture of polymeric nucleus nanoparticles: one involves the consecutive mixture of the nucleus and hull structure, and the other involves the self-assembling of block copolymers.

\section{Related Works}

Industrial production can be scaled using macromolecular self-assembly techniques in determining the transformation of unique polymer core-shell nanoparticles. The characterization, application, and synthesis of polymeric nanoparticles were proposed by Wang et al. [5]. This work describes the methods of assembly and algorithms that regulate the proposal of mechanical properties required for favorable use, such as improving the performance of the extract in elastic vulcanization and improving the performance of rubber configurations. The nanoparticles were made by polymerizing block copolymers and allowing them to self-assemble into micelles in solvents, followed by core cross-linking to stabilize their structure. A range of coreshell nanoparticles of various forms are produced which are dependent on the kind and macrostructure of the block copolymers, the concentration, the solvent, and other progression factors. The majority of the nanoparticles had a solid, elastomeric, and deeply cross-linked core shell. The progression and structure of nanoparticles have been studied and characterized throughout different phases of the process.

PNPs are particle dispersions or solid particles ranging in size from 10 to 1000 nanometers. The use of particle delivery schemes as carriers for small and large molecules has flashed a lot of investigations in the field of medicine delivery. Nagavarma et al. [6] proposed a review on different methods utilized in the polymeric nanoparticles' preparations. Particulate systems, such as nanoparticles, have been used to modify and enhance the pharmacokinetics and pharmacodynamics of many medicinal compounds. Several approaches for generating polymeric nanoparticles have been established, and these methods are categorized based on the particle production which involves a reaction in polymerization or whether the nanoparticles form directly from a macromolecule or premade polymer. Many strategies for preparing polymeric nanoparticles are explained in this review.

A review on the drug-laden polymer nanoparticle is done based on the strategies employed in modern medicine. The method was proposed by Castro et al. [7], formulation capable of delivering medication in a regulated and precise manner at a given location. In this situation, stimulus-reactive nanoparticles are identified because they have lengthy circulation durations, can reach the illness site, and can optimize intracellular medicine administration. As a result, this review focused on current trends in drug delivery techniques as well as the impact of external and internal stimulus causes on stimulus-reactive nanocarriers. In addition, both traditional and novel approaches for the manufacture of polymer nanomaterials were examined.

The polymeric nanoparticles were produced as aqueous distributions using an emulsion that included a salting step to remove surfactants and chlorinated solvents. The preparation of the aqueous polymeric nanodispersion method was proposed by Allémann et al. [8]; this was done by a changeable salting-out procedure, and the particle size was influenced by this process. A salting-out technique prevents acetone from combining with water in the saturated aqueous solution. Following the production of emulsion of oil-inwater, an adequate amount of water is added to allow complete acetone dispersal into the aqueous phase, resulting in the development of nanospheres. During the production process, the stirring rate, internal/external phase ratio, 
polymer absorption in the acetone phase, electrolyte type, and attentiveness and type of PVAL in the aqueous stage were all modified.

\section{Methodology}

For the production of numerous and different molecular designs, the polymer is found to be a convenient material. These molecular designs could be integrated into an inimitable nanoparticle, and they are used to design different medical applications. For the preparation of polymeric nanoparticles, more than a few methods were developed during the last two decades. Based on their information, the design procedure of the PNP can be classified, and this includes the reaction of polymerization or formation of the nanoparticle from the macromolecule, and the ionic gelation method is also included [9]. Based on the preparation of the nanoparticle, nanocapsules or nanospheres are obtained. Figure 1 shows the difference in the nanosphere and nanocapsule. The drugs confined in the cavity are defined as nanocapsules, and the cavity is enclosed by an inimitable polymer membrane; on the contrary, the atmosphere system in which the drug is substantially and unvaryingly dispersed is termed as the nanosphere. Polymer nanoparticles play an important role in the field of electronics, conducting materials, photonics, medicine, environmental technology of the sensor system, and pollution control.

\section{Nanoparticle Preparation and the Polymer}

The polymers should be adaptable (nontoxic) and antigenicity-free, as well as biodegradable and biocompatible with the human body. The natural polymer and the synthetic polymer used in the preparation of polymeric nanoparticles are given in Table 1.

Depending on the particular application, the assets of the polymer nanoparticle could be optimized. The method on the preparation plays a dynamic role in achieving properties of the PNP. As a result, having preparation methods to create PNPs with the appropriate characteristics for a certain application is quite beneficial. Polymerization, ionic gelation, and premade polymers are among the procedures employed [10]. Appropriate polymer nanoparticle is designed based on the desired properties for specific applications, and this can be done during the preparation of the polymerization of monomers. In this research, two important methods were discussed based on the preparation of polymeric nanoparticles. They are emulsion polymerization and salting out [11].

\subsection{Method Used in Polymeric Nanoparticle Preparation.} There are different methods used in the polymeric nanoparticle preparation, and they are expressed in Figure 2(a) which shows the dispersion of the performed polymer, and Figure 2(b) shows the polymerization of monomers. At first, the preparation of the nanoparticle from the dispersion of the performed polymer is presented, and then the nanoparticle preparation from the polymerization of monomers is expressed [12].

\section{Preparation of the Nanoparticle from the Polymerization of the Monomer}

5.1. Emulsion Polymerization. The fastest method of polymeric nanoparticle preparation is the emulsion polymerization method, and it is readily ascendable. Based on the organic or aqueous phase, the following method is divided into two categories. The dispersion of the monomer into an emulsion is involved in the continuous organic phase; this can also be defined as the inverse microemulsion or nonsolvent monomers [13]. To avoid aggregation at the beginning of polymerization, protective or surface-active soluble polymers were used as a method of producing the nanoparticle. Due to its requirements, this method is said to be of low impact; the requirements include the organic toxic solvent, monomers, mediators, and surfactants. These parameters are neglected from the nanoparticles prepared [14]. The alternative method provides more interest when the preparation process becomes difficult to handle, and as a result, the nature of the polymer becomes nonbiodegradable. By the dispersion process, nanoparticles such as polymethyl methacrylate, poly-ethyl cyanoacrylate, and polybutyl cyanoacrylate were prepared, and in this process, the organic phase used solvents such as cyclohexane, pentane, and toluene. In the aqueous continuous phase, emulsifier or surfactants are not essential since in the continuous phase situation, the monomers dissolved, and this is commonly used as an aqueous solution.

Figure 3 illustrates the emulsification process. In different mechanism processes, the polymerization process could be originated. When the monomer molecule gets dissolved in the continuous phase, the initiator molecule strikes with it. The originator molecule may be ions or radicals. The monomer molecule gets transformed into originating radicals by the high-energy radiation system, and this includes g-radiation or UV rays and strong visible light [15]. A mechanism of anionic polymerization causes monomeric ions or monomeric radicals to collide with other monomeric molecules, causing the chain to develop. Stage parting and the creation of solid particles may occur before or after the completion of the polymerization reaction.

5.2. Miniemulsion Polymerization. In recent years, the number of articles published on polymerization based on miniemulsion and the creation of a wide range of useful polymer materials has improved substantially. Stabilizers, water, monomer blends, surfactants, and initiators are common ingredients in miniemulsion polymerization formulations. The common parameters of the polymerization in miniemulsion are monomer mixture, water, stabilizer, and originator; these were used based on the formulization. The use of a molecule with a low molecular mass as a costabilizer [16], as well as the use of a high-shear device, distinguishes polymerization emulsion from polymerization miniemulsion. Lot of interfacial tension was produced in the miniemulsion process, and to attain the steady-state condition, a great shear is required, and the miniemulsion is critically stabilized. 


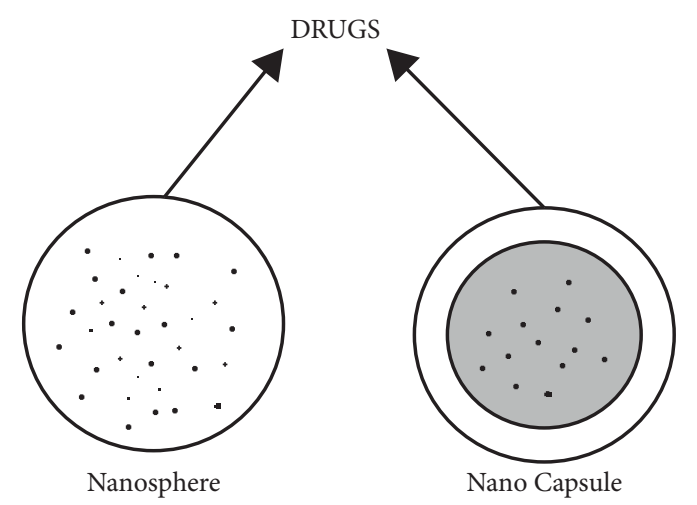

Figure 1: Difference in the nanosphere and nanocapsule.

TABle 1: Classification of polymers.

\begin{tabular}{lcc}
\hline No. & Natural polymer & Synthetic polymer \\
\hline 1 & Chitosan & Polylactides \\
2 & Gelatin & Polyglycolides \\
3 & Sodium alginate & PLGA (polylactide-co-glycolide) \\
4 & Albumin & Polyanhydrides \\
\hline
\end{tabular}

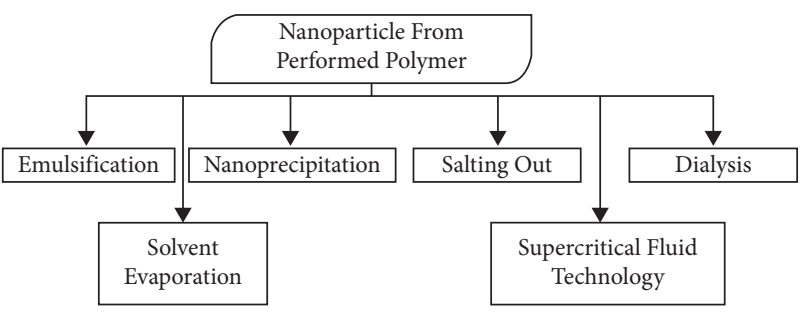

(a)

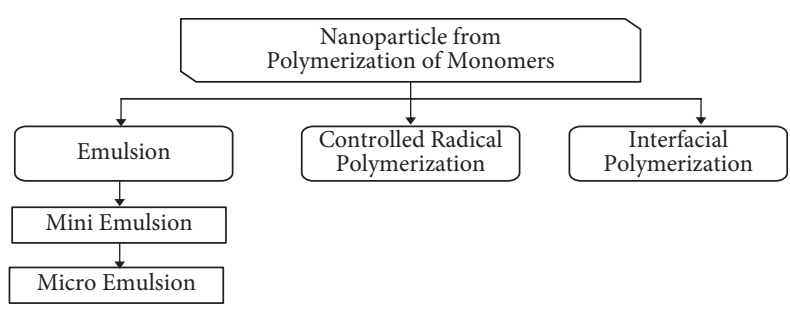

(b)

Figure 2: Polymerization of monomers.

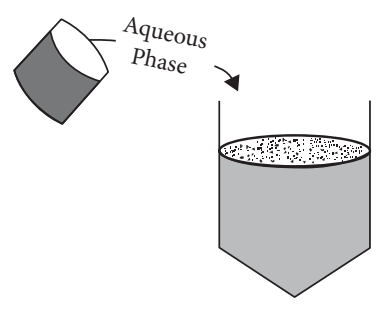

Oil Phase

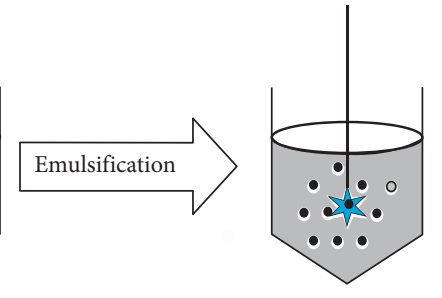

Water in oil emulsion

FIGURE 3: Emulsification process.

5.3. Microemulsion Polymerization. Microemulsion polymerization is a successful method to produce nanosized polymer particles which has received much attention. In spite of the fact that emulsion and polymerization by microemulsion can form colloidal polymer particles with a large molar mass, they are completely different kinetically. The particle size and average number of strings per particle are substantially smaller in microemulsion polymerization. An initiator, often water soluble, is added to the aqueous phase of a stable thermodynamic microemulsion containing inflated micelle in microemulsion polymerization. The polymerization process begins with this steady, spontaneously generated thermodynamic state and is based on large quantities of surfactants with low interfacial tension at the oil/water interface. Furthermore, due to the application of a large quantity of the surfactant, the particles are completely coated with the surfactant. Subsequently, the osmotic and elastic impact of the chains destabilized fragile microemulsions, causing particles to grow, empty micelles to form, and secondary nucleation. Some of the critical elements 
determining microemulsion polymerization kinetics and PNP characteristics include the initiator type and concentration, surfactants, monomer, and reaction temperature.

\subsection{Determining the Molecular Weight and Intrinsic Viscosity} of the Polymeric Nanoparticle. By using the Ubbelohde capillary viscometry method, the molecular weights of the final polymers could be determined. Equation (1) determines that the polyacrylamide nanoparticle at $25^{\circ} \mathrm{C}$ is dissolved in water.

$$
(\zeta)_{\text {intrinsic }}\left(\frac{\mathrm{ml}}{\mathrm{g}}\right)=6.31 \times 10^{-3} M_{w}^{0.8}\left(\frac{\mathrm{g}}{\mathrm{mol}}\right) .
$$

Excess of acetone $(1: 6)$ was used to precipitate a piece of each final emulsion, which was then washed multiple times with acetone. The precipitates were then dried for 48 hours at $60^{\circ} \mathrm{C}$. Under stirring, 200 milligrams of pure polymer powder were dissolved in 100 milliliters of deionized water. The produced solution $(0.2 \mathrm{~g}$ of polymer per $100 \mathrm{~mL}$ ) was diluted to provide concentrations of $0.18,0.14,0.10$, and $0.06 \mathrm{~g}$ of the polymer per $100 \mathrm{~mL}$ deionized water. A capillary viscometer (constant factor $=0.01026 \mathrm{~mm}^{2} / \mathrm{s}$ ) was used to measure the viscosity of each solution three times. The intrinsic viscosity is calculated from the intercept value of a linear regression, and this has a link with the specific viscosity and polymer content. The molecular weight of the finished polymer is determined using the DLS method.

5.5. Measurement of Monomer Conversion. $15 \mathrm{~g}$ of dispersion $\left(n_{1}\right)$ was removed from the reaction vessel at various time intervals to determine AM conversion as a function of time. To stop the polymerization process, each sample was given a $0.10 \mathrm{wt} \%$ hydroquinone aqueous solution. To achieve constant weights, samples were precipitated with excess of acetone $(1: 8)$ and dried in a freeze dryer at $50^{\circ} \mathrm{C}$ for 48 hours $\left(n_{2}\right)$. Finally, equation (2) was used to compute the conversion of PAM nanoparticles.

$$
y \%=\frac{n_{2}-\text { hydroquinone weight }}{\text { primary monomer weight/weight of the particle based on the parameter }} \times 100 \% \text {. }
$$

5.6. Size and Distribution of the Emulsion Particle. By using the Malvern Zetasizer Nano series, the $Z$-average diameter of the polyacrylamide nanoparticle is determined, and the distribution is measured. Under the scattering angle of $90^{\circ}$, the light source with a wavelength of $645 \mathrm{~nm}$ is measured, and this has an ambient temperature of $30^{\circ} \mathrm{C}$. After vigorous mixing, the emulsions were injected into $10 \%$ Span 80 in cyclohexane solution $(10,000 \mathrm{rpm})$. A field emission scanning electron microscope is used to measure particle size. On the sample holder, one drop of the finished emulsion was deposited without any treatment and then spin-coated to remove water. It is worth noting that the $Z$-average employed an intensity-based estimated value of particle size which is the optimum value to provide for particle size in the face of even modest changes in the sample in dynamic light scattering studies.

\section{Results and Discussion}

There are various methods involved in the preparation of nanoparticles, and different technologies have been evolved for the preparation of the nanoparticle from the polymeric material. To prepare the nanoparticle from the polymeric material, a simple, safe, and reproducible method has been adapted; now, this type of method is used in the preparation of the drug-loaded nanosphere and nanocapsules [17]. Various polymeric nanoparticle preparation processes are available. It is discovered that formulating PNPs is a cutting-edge skill that necessitates the selection of an appropriate procedure from a variety of options. Three characteristics distinguish nanoparticle preparation methods: (1) reagents that are less hazardous are required, (2) procedure simplification to allow for costeffective scale-up, and (3) to improve the yield and entrapment efficiency by optimization. The general advantage and disadvantage about the preparation of nanoparticles are defined [18]. Polymeric nanoparticles design experiment is represented in Table 2. These values ensure that the results were consistent. Experimental errors for monomer conversion, intrinsic viscosity and particle size, molecular weight and distribution, and second virial coefficient were found to be $0.2 \%, 1 \%, 1.5 \%$, and $3 \%$, respectively.

Figure 4 shows the polymeric nanoparticle design experiment which is illustrated based on Table 2. The graph is plotted for the water and oil ratio, HLB, water, and $\mathrm{CH}$ at wbt\%. From the graph, it can be verified that the level of water and $\mathrm{CH}$ get increased after a constant range and again maintained a constant range.

6.1. Temperature and Reaction Time Effects. Table 3 represents the full conversion of monomers. When a portion of the emulsion was taken from the reaction vessel at different time intervals, six reactions were evaluated: 20 minutes, 125 minutes, and 210 minutes. After 120 minutes, the results were stable, revealing the time required for nearly full monomer conversion. In the subsequent runs, a polymerization time of 120 minutes was employed. The droplet phase increased the radical desorption rate of initiator-derived radicals (as the water-soluble APS initiator was used) due to the reduced average number of radicals per particle (the expected value for the examined system at $60^{\circ} \mathrm{C}$ is substantially below 0.5 ), and the resulting polymers will have a higher molecular weight and intrinsic viscosity. 
TABle 2: Polymeric nanoparticle design experiment.

\begin{tabular}{|c|c|c|c|c|c|c|c|}
\hline Run & Initiator & $\mathrm{W} / \mathrm{O}$ ratio & HLB & Water (wbt\%) & $\mathrm{CH}(\mathrm{wbt} \%)$ & $T\left({ }^{\circ} \mathrm{C}\right)$ & $N(\mathrm{rpm})$ \\
\hline 1 & APS & 0.1 & 6.8 & 5.2 & 89.9 & 65 & 550 \\
\hline 2 & APS & 0.2 & 6.8 & 11.4 & 81.8 & 65 & 550 \\
\hline 3 & APS & 0.3 & 6.8 & 15.0 & 76.2 & 65 & 550 \\
\hline 4 & APS & 0.5 & 6.8 & 34.9 & 76.2 & 65 & 550 \\
\hline 5 & APS & 0.4 & 6.8 & 16.5 & 76.2 & 65 & 550 \\
\hline 6 & APS & 0.4 & 6.8 & 16.5 & 76.2 & 65 & 550 \\
\hline 7 & APS & 0.4 & 6.8 & 16.5 & 76.5 & 65 & 550 \\
\hline 8 & APS & 0.4 & 6.8 & 16.5 & 76.8 & 65 & 550 \\
\hline 9 & APS & 0.4 & 6.8 & 16.5 & 76.2 & 65 & 550 \\
\hline 10 & APS & 0.4 & 6.8 & 16.5 & 76.9 & 65 & 550 \\
\hline 11 & APS & 0.4 & 6.8 & 16.5 & 76.7 & 65 & 550 \\
\hline
\end{tabular}

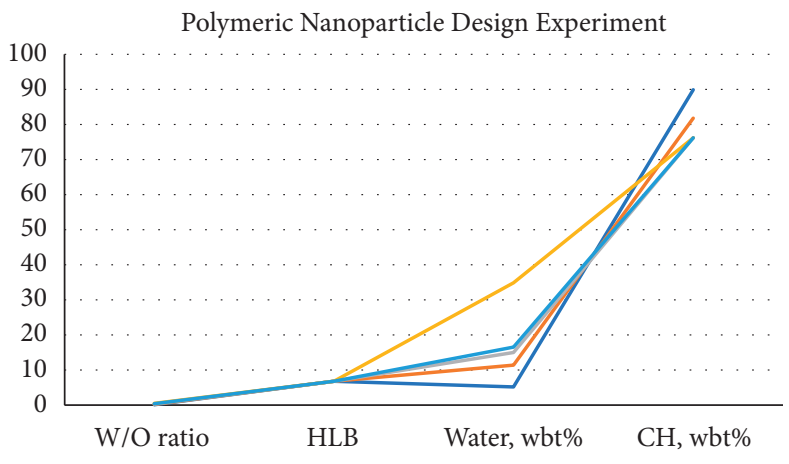

FIgURe 4: Polymeric nanoparticle design experiment.

TABle 3: Effect of time and temperature.

\begin{tabular}{|c|c|c|c|c|c|c|c|c|c|c|}
\hline \multirow[t]{2}{*}{ Run } & \multirow[t]{2}{*}{ Time } & \multirow[t]{2}{*}{ Temperature } & \multicolumn{4}{|c|}{ Technique of dynamic light scattering } & \multicolumn{2}{|c|}{$\begin{array}{c}\text { Capillary viscometer } \\
\text { technique }\end{array}$} & \multirow{2}{*}{$M_{w}^{\mathrm{VDif}}$} & \multirow[t]{2}{*}{$Y \%$} \\
\hline & & & $D(\mathrm{~nm})$ & PSD & $M_{w}^{\mathrm{DL}}(\mathrm{MDA})$ & $A(\mathrm{~mL} \mathrm{~mol})$ & {$[\zeta](\mathrm{mL} / \mathrm{g})$} & $M_{w}^{\mathrm{VS}}$ & & \\
\hline $3^{\prime}$ & 25 & 60 & 65 & $64-165$ & 0.88 & $+0.205 E-5$ & 345 & 0.85 & 3.46 & 68.0 \\
\hline 3 & 125 & 0 & 60 & $50-180$ & 6.55 & $+0.670 E-4$ & 1675 & 6.05 & 7.88 & 99.2 \\
\hline $3^{\prime \prime}$ & 250 & 0 & 61 & $28-160$ & 6.55 & $+0.655 E-5$ & 1678 & 6.04 & 7.25 & 99.9 \\
\hline 6 & 125 & 55 & 65 & $45-189$ & 5.15 & $+0.685 E-4$ & 1470 & 5.15 & 1.05 & 85.8 \\
\hline 5 & 125 & 45 & 65 & $50-220$ & 4.05 & $+0.680 E-4$ & 1210 & 4.00 & 0.30 & 59.5 \\
\hline
\end{tabular}

As a result, as the temperature rises, the ratio of propagation to termination constant will rise, as will the length of polymer chains and their intrinsic thicknesses, under the investigated conditions (Table 3 ). The reaction temperature had no effect on the stability of the latex dispersions because the $A_{2}$ coefficients were not significantly different.

Figure 5 shows the variation of monomers which is represented in a graphical form. It is obvious that increasing the reaction temperature increased polymerization rate, resulting in a significant increase in conversion over the entire reaction time, with nearly full conversion reached in only 125 minutes in the reaction done at $65^{\circ} \mathrm{C}$. As a result, the polymer chains' length and their inherent viscosities will grow under the examined conditions, as would the ratio of propagation to termination constant at improved temperature.

Figure 6 shows the W/O reaction, which depicts the kinetics of inverse emulsion polymerization of AM using various initiators. The reaction rate for the system begun using APS is clearly higher than that for the system initiated with AIBN.

Figure 7 represents different initiator types where PSD and SEM pictures of nanoparticles generated at various agitation rates are displayed. As the initiator concentration is increased, the molecular weight of the resultant polymer drops because of higher number of initiators produced radicals inside similar size particles.

The effect of the polymer in nanoparticles on loading and entrapment efficiency is given in Table 4, and it is illustrated in Figure 8. The values of loading efficiency are in the range of $7.95-16.8 \%$, and the values of entrapment efficiency are about $56.7-74.8 \%$. It is predicted that entrapment efficiency is high for the formulation of polymer nanoparticles. The loading efficiency is improved by improving the polymer ratio so that the adequate quantity of the polymer will be available to entrap the drug present 


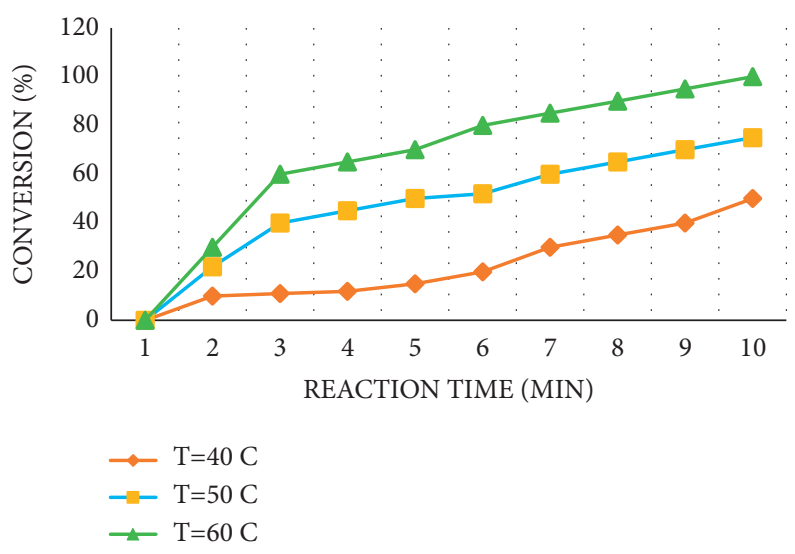

Figure 5: Variation of monomers.

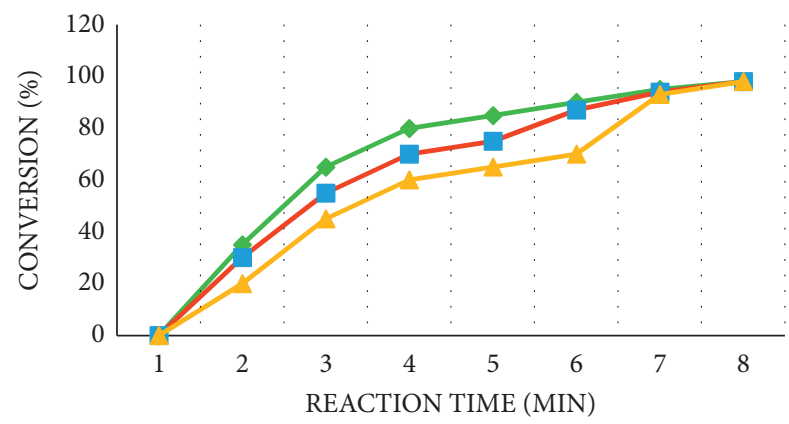

$$
\begin{aligned}
& \rightarrow \mathrm{W} / \mathrm{O}=0.2 \\
& \rightarrow \mathrm{W} / \mathrm{O}=0.3 \\
& \multimap \mathrm{W} / \mathrm{O}=0.5
\end{aligned}
$$

Figure 6: W/O reaction.

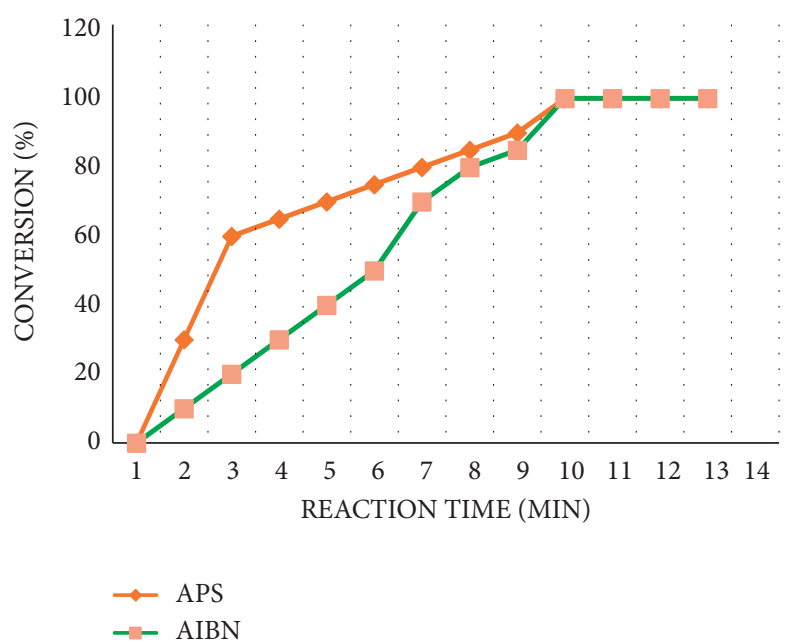

FIgURE 7: Different initiator types. 
TABLE 4: Loading and entrapment efficiency.

\begin{tabular}{lccc}
\hline Sl. no. & Formulation code & Loading efficiency & Entrapment efficiency \\
\hline 1 & PNP 1 & 12.32 & 56.7 \\
2 & PNP 2 & 16.8 & 72.7 \\
3 & PNP 3 & 7.95 & 74.8 \\
\hline
\end{tabular}

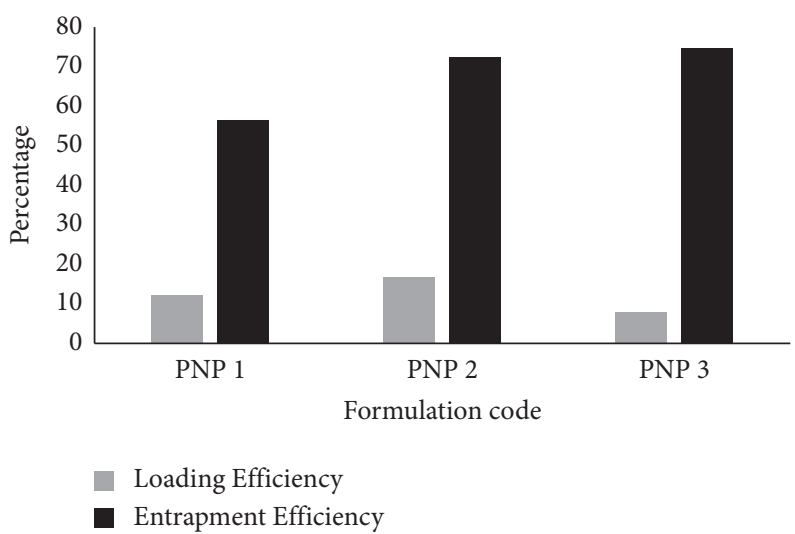

FIGURE 8: Effect on the loading and entrapment efficiency of the polymer.

in the solution. The polymer may be labeled or grafted onto the polymer by integrating it during the manufacturing process of nanoparticles made from premade polymers. PCL and PLA, for example, can determine the concentration after using a fast technique that occurs in organic resolution at low temperatures and may be used on polymers with high molecular weight.

\section{Conclusion}

The research was designed to provide the details about the preparation of nanoparticles. There are different methods used in preparing the polymeric nanoparticles. Polymerases, which can be found in the field of polymer colloids, are new structures awaiting to join the family of nanoparticulate drug delivery devices. Most of the polymeric nanoparticles are used mainly in the preparation of the drugs in the medical field such as nanospheres and nanocapsules. To describe the relation to nanospheres, different types of drugs are associated. These drugs may dissolve within the polymeric matrix or get absorbed by the polymer. The effect of nanoparticles based on the temperature and time is discussed in the result section. The research expresses the details about the preparation of polymeric nanoparticles from different polymerization methods. Salting-out and emulsification methods are expressed with the diagrammatic representation, and finally, the advantages and drawbacks about the nanoparticle preparation method are tabulated. Thus, the novel approach for the preparation of polymer nanoparticles is presented.

\section{Data Availability}

The data used to support the findings of this study are included within the article.

\section{Conflicts of Interest}

The authors declare that there are no conflicts of interest regarding the publication of this article.

\section{Acknowledgments}

The authors would like to express their gratitude towards Vignan's Foundation for Science, Technology and Research (Deemed to be University) for providing the necessary infrastructure to carry out this work successfully.

\section{References}

[1] S. Sahoo, "Conduction and switching behavior of e-beam deposited polycrystalline $\mathrm{Nb}_{2} \mathrm{O}_{5}$ based nano-ionic memristor for non-volatile memory applications," Journal of Alloys and Compounds, vol. 866, Article ID 158394, 2021.

[2] M. Joshi and U. Chatterjee, "Polymer nanocomposite," in Advanced Composite Materials for Aerospace EngineeringElsevier, Amsterdam, Netherlands, 2016.

[3] S. Sahoo, P. Manoravi, and S. R. S. Prabaharan, "Titania based nano-ionic memristive crossbar arrays: fabrication and resistive switching characteristics," Nanoscience and Nanotechnology-Asia, vol. 9, no. 4, pp. 486-493, 2019.

[4] E. M. Pridgen, F. Alexis, and O. C. Farokhzad, "Polymeric nanoparticle drug delivery technologies for oral delivery applications," Expert Opinion on Drug Delivery, vol. 12, no. 9, pp. 1459-1473, 2015.

[5] X. Wang, J. E. Hall, S. Warren et al., "Synthesis, characterization, and application of novel polymeric nanoparticles," Macromolecules, vol. 40, no. 3, pp. 499-508, 2007.

[6] B. Nagavarma, H. K. Yadav, A. Ayaz, L. Vasudha, and H. Shivakumar, "Different techniques for preparation of polymeric nanoparticles-a review," Asian Journal of Pharmaceutical and Clinical Research, vol. 5, no. 3, pp. 16-23, 2012.

[7] K. C. d. Castro, J. M. Costa, and M. G. N. Campos, "Drugloaded polymeric nanoparticles: a review," International 
Journal of Polymeric Materials and Polymeric Biomaterials, vol. 2020, Article ID 1798436, 13 pages, 2020.

[8] E. Allémann, R. Gurny, and E. Doelker, "Preparation of aqueous polymeric nanodispersions by a reversible salting-out process: influence of process parameters on particle size," International Journal of Pharmaceutics, vol. 87, no. 1-3, pp. 247-253, 1992.

[9] S. Moein Moghimi, "Recent developments in polymeric nanoparticle engineering and their applications in experimental and clinical oncology," Anti-Cancer Agents in Medicinal Chemistry, vol. 6, no. 6, pp. 553-561, 2006.

[10] V. Vijayan, K. R. Reddy, S. Sakthivel, and C. Swetha, "Optimization and charaterization of repaglinide biodegradable polymeric nanoparticle loaded transdermal patchs: in vitro and in vivo studies," Colloids and Surfaces B: Biointerfaces, vol. 111, pp. 150-155, 2013.

[11] S. Mallakpour and V. Behranvand, "Polymeric nanoparticles: recent development in synthesis and application," Express Polymer Letters, vol. 10, no. 11, pp. 895-913, 2016.

[12] J. Liu, Z. Qiu, S. Wang, L. Zhou, and S. Zhang, "A modified double-emulsion method for the preparation of daunorubicin-loaded polymeric nanoparticle with enhanced in vitro anti-tumor activity," Biomedical Materials, vol. 5, no. 6, Article ID 065002, 2010.

[13] C. Vauthier and K. Bouchemal, "Methods for the preparation and manufacture of polymeric nanoparticles," Pharmaceutical Research, vol. 26, no. 5, pp. 1025-1058, 2009.

[14] C. Zhao and X. Li, "Synthesis of high solid content (ST/BA/ AA) emulsion, particle size and its distribution," Polymeric Materials: Science and Engineering, vol. 2, 2005.

[15] C. Pinto Reis, R. J. Neufeld, A. J. Ribeiro, and F. Veiga, "Nanoencapsulation I: methods for preparation of drugloaded polymeric nanoparticles," Nanomedicine: Nanotechnology, Biology and Medicine, vol. 2, no. 1, pp. 8-21, 2006.

[16] A. J. Ribeiro, "Chapter 7: preparation of drug-loaded polymeric nanoparticles," in Nanomedicine in Cancer, pp. 171214, Jenny Stanford Publishing, Dubai, UAE, 2017.

[17] B. N. Ganguly, Nanomaterials in Bio-Medical Applications: A Novel Approach, Materials Research Foundations, Millersville, PA, USA, 2018.

[18] R. Karnik, F. Gu, P. Basto et al., "Microfluidic platform for controlled synthesis of polymeric nanoparticles," Nano Letters, vol. 8, no. 9, pp. 2906-2912, 2008. 